\begin{tabular}{|c|c|}
\hline Title & $\begin{array}{l}\text { POLYFLUOROALKYLATION OF CARBONYL COMPOUNDSBY POLYFLUOROALKYL ANIONS } \\
\text { GENERATED FROM POLYFLUOROCARBOXAMIDES }\end{array}$ \\
\hline Author(s) & Wakita, Natshumi; Hara, Shoji \\
\hline Citation & $\begin{array}{l}\text { Heterocycles, 88(2), 1201-1212 } \\
\text { https://doi.org/10.3987/COM-13-s(S)84 }\end{array}$ \\
\hline Issue Date & 2014-01-27 \\
\hline Doc URL & http:/hdl.handle.net/2115/54926 \\
\hline Type & article (author version) \\
\hline File Information & $\begin{array}{l}\text { POLYFLUOROALKYLATION OF CARBONYL COMPOUNDSBY POLYFLUOROALKYL ANIONS } \\
\text { GENERATED FROM POLYFLUOROCARBOXAMIDES.pdf }\end{array}$ \\
\hline
\end{tabular}

Instructions for use 


\title{
POLYFLUOROALKYLATION OF CARBONYL COMPOUNDS BY POLYFLUOROALKYL ANIONS GENERATED FROM POLYFLUOROCARBOXAMIDES
}

\author{
Natshumi Wakita and Shoji Hara*
}

Graduate School of Engineering, Hokkaido University, Sapporo 060-8628, Japan E-mail: shara@eng.hokudai.ac.jp

\begin{abstract}
Polyfluoroalkyl anions, generated by reduction of (polyfluoroalkanoyl)piperidines with $\mathrm{Et}_{3} \mathrm{BHK}$, were used for the polyfluoroalkylation of carbonyl compounds. Trifluoromethylation of aromatic aldehydes proceeded in good yields, and that of aliphatic aldehydes afforded a moderate yield. In contrast, the yield was low when the reaction involved benzophenone. Pentafluoroethylation and octafluorobutylation of aldehydes were also carried out by using the corresponding (polyfluoroalkanoyl)piperidines, which were prepared from commercially available polyfluorocompounds. The (polyfluoroalkanoyl)piperidines were also prepared through polyfluorination, and were used in the polyfluoroalkylation of aldehydes.
\end{abstract}

\section{INTRODUCTION}

Nucleophilic trifluoromethylation of carbonyl compounds has been widely performed to introduce a trifluoromethyl group into the substrate. ${ }^{1}$ The trifluoromethyl anion is unstable. Therefore, it is generated from the precursor in the presence of an electrophile such as a carbonyl compound. Although a number of various trifluoromethyl compounds have been used as precursors, trifluoromethyltrimethylsilane has been most frequently used and is well-studied.1c,1d,2 Recently, trifluoroacetaldehyde hemiaminals (1) have attracted much attention as an accessible trifluoromethyl source, because they can be prepared from economical materials such as fluoroform ${ }^{1 \mathrm{j}, 3}$ and trifluoroacetaldehyde hemiacetal. ${ }^{1 \mathrm{j}, 4}$ They are converted to their metal salts (2) to generate the trifluoromethyl anion. ${ }^{5}$ We can obtain 2 by the reduction of trifluoroacetamide (3). ${ }^{6}$ Moreover, various (polyfluoroalkanoyl)amides are obtainable from either commercially available 
polyfluorocompounds or through polyfluorination reactions, and they can be used as a source for polyfluoroalkyl anions (Scheme 1).

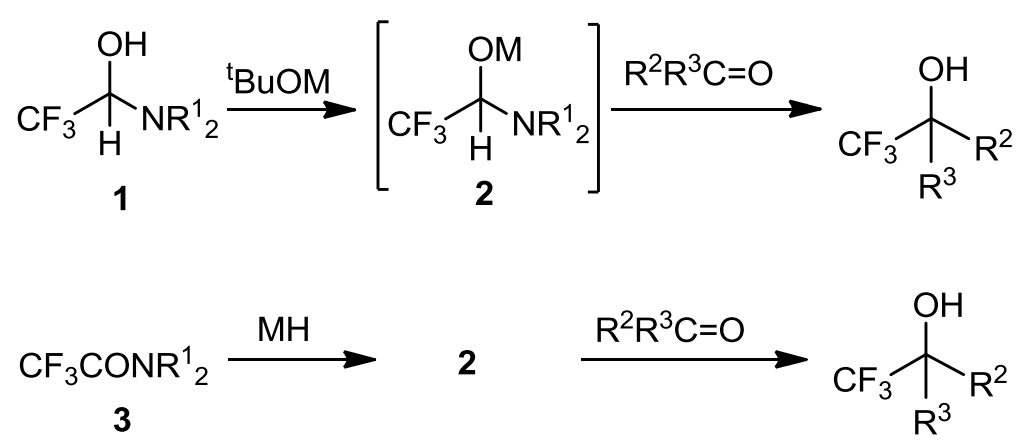

Scheme 1

\section{RESULTS AND DISCUSSION}

Trifluoromethylation of benzaldehyde was carried out using $N$-(trifluoroacetyl)piperidine, $N$-(trifluoroacetyl)pyrrolidine, and $N, N$-diethyl trifluoroacetamide as trifluoromethyl anion sources, and potassium triethylborohydride, DIBAH and potassium triisopropoxyborohydride as reducing reagents under various conditions (Table 1). A reducing reagent and benzaldehyde were successively added to the amide in THF at $-78{ }^{\circ} \mathrm{C}$ (Entries 1-5) or at room temperature (Entry 6), and the resulting mixture was stirred for $24 \mathrm{~h}$ at $50{ }^{\circ} \mathrm{C}$. Maintaining an interval between each addition of the reagents did not improve the results. The expected trifluoromethylated product (2a) was formed, only when $N$-(trifluoroacetyl)piperidine or $N$-(trifluoroacetyl)pyrrolidine was treated with potassium triethylborohydride (Entries 1, 4, and 6). The best yield was obtained by adding potassium triethylborohydride and benzaldehyde to $N$-(trifluoroacetyl)piperidine at room temperature (Entry 6). 
Table 1. Trifluoromethylation of benzaldehyde under various conditions ${ }^{a}$

\begin{tabular}{|c|c|c|c|}
\hline Entry & $\mathrm{NR}_{2}$ & $\mathrm{MH}$ & Yield $(\%)^{b}$ \\
\hline 1 & & $\mathrm{KBEt}_{3} \mathrm{H}$ & 57 \\
\hline 2 & & DIBAH & 0 \\
\hline 3 & & $\mathrm{~KB}(\mathrm{OPr}-\mathrm{i})_{3}$ & 0 \\
\hline 4 & & $\mathrm{KBEt}_{3} \mathrm{H}$ & 47 \\
\hline 5 & $\mathrm{NEt}_{2}$ & $\mathrm{KBEt}_{3} \mathrm{H}$ & 0 \\
\hline 6 & $y^{\prime}$ & $\mathrm{KBEt}_{3} \mathrm{H}$ & $99^{c}$ \\
\hline
\end{tabular}

a. If otherwise not mentioned, 2 eq of the amide and $\mathrm{MH}$ to benzaldehyde were used. The reagents were added at $-78^{\circ} \mathrm{C}$, and the mixture was stirred at $50{ }^{\circ} \mathrm{C}$ for $24 \mathrm{~h}$. b. ${ }^{19} \mathrm{~F}$ NMR yield based on benzaldehyde.

c. The reagents were added at room temperature.

The present trifluoromethylation reaction was applied to various carbonyl compounds. In the reaction with aromatic aldehydes and cinnamaldehyde, the corresponding trifluoromethylated products were obtained in good yield (Entries 1-5 in Table 2). From adamantane-1-carbaldehyde that has no $\alpha$-proton, the trifluoromethylated product was obtained in $60 \%$ yield (Entry 6). In contrast, the reaction with cyclohexanecarbaldehyde that has $\alpha$-proton, afforded an aldol reaction product as the main product. However, when the reaction was carried out in $\mathrm{CH}_{2} \mathrm{Cl}_{2}$ instead of THF, the trifluoromethylated product was obtained in $45 \%$ yield (Entry 7). In the reaction with benzophenone, most of the starting material remained unchanged (Entry 8). 
Table 2. Trifluoromethylation of carbonyl compounds ${ }^{a}$<smiles>O=C(N1CCCCC1)C(F)(F)F</smiles><smiles>[R20]C(=O)OCC(C)C</smiles><smiles>[R]C([R])(O)C(F)(F)F</smiles>

Entry Carbonyl compound

a. If otherwise not mentioned, the reaction was carried out in THF using 2 eq of $\mathrm{KBEt}_{3} \mathrm{H}$ and amide to carbonyl compound. The reagents were mixed at room temperature and stirred at $50^{\circ} \mathrm{C}$ for $24 \mathrm{~h}$. b. Isolated yield base on carbonyl compound used. In parentheses, ${ }^{19} \mathrm{FNMR}$ yield. c. $\mathrm{CH}_{2} \mathrm{Cl}_{2}$ was used as solvent 
As various (polyfluoroalkanoyl)piperidines are obtainable from commercially available polyfluorocompounds, the present method is applicable to the various polyfluoroalkylation of carbonyl compounds. For example, $N$-(pentafluoropropanoyl)piperidine (1b) was prepared from methyl pentafluoropropionate ${ }^{7}$ and was used for the pentafluoroethylation of 1-naphthaldehyde. The reaction proceeded similar to trifluoromethylation, and the pentafluoroethylated product (2i) was obtained in high yield. $N$-(2,2,3,3,4,4,5,5-Octafluoropetanoyl)piperidine (1c) was prepared from commercially available 2,2,3,3,4,4,5,5-octafluoropentanol in three steps $^{7-9}$ and was used in the reaction with 1-naphthaldehyde. The reaction of $\mathbf{1 c}$ with aldehyde was sluggish and the octafluorobutylated product $\mathbf{2 \mathbf { j }}$ was formed in poor yield under the trifluoromethylation conditions. However, $\mathbf{2} \mathbf{j}$ was obtained in $61 \%$ yield by carrying the reaction at $40{ }^{\circ} \mathrm{C}$ for $24 \mathrm{~h}$ using 4 equivalents of $\mathbf{1 c}$ and $\mathrm{KBEt}_{3} \mathrm{H}$ to 1-naphthaldehyde without solvent (Scheme 2).<smiles>O=C(N1CCCCC1)C(F)(F)F</smiles>

$1 b$

1) $\mathrm{KBEt}_{3} \mathrm{H}$<smiles>O=C(N1CCCCC1)C(F)(F)F</smiles>

$1 \mathrm{c}$<smiles>CCCC(C)C(O)c1cccc2ccccc12</smiles>

2)<smiles>CCCC(C)C(=O)c1cccc2ccccc12</smiles><smiles>OC(c1cccc2ccccc12)C(F)(F)C(F)(F)F</smiles>

2i
2)<smiles>O=Cc1cccc2ccccc12</smiles><smiles>OC(c1cccc2ccccc12)C(F)(F)F</smiles>

Scheme 2

(Polyfluoroalkanoyl)piperidines can be also prepared through polyfluorination reactions. For example, methyl 2-(arylsulfanyl)-2,2-difluoroacetate (3) was prepared by difluorination of methyl 2-(arylsulfanyl)acetate using $\mathrm{IF}_{5}-\mathrm{Et}_{3} \mathrm{~N}-3 \mathrm{HF},{ }^{10}$ and then converted to $N$-2-(arylsulfanyl)-2,2-difluoroacetylpiperidine (4). ${ }^{7}$ The reaction of $\mathbf{4}$ with an aldehyde was performed as in the case of $\mathbf{2 a}$, and the (arylsulfanyl)difluoromethylated product (5) was obtained in high yield (Scheme 3). 


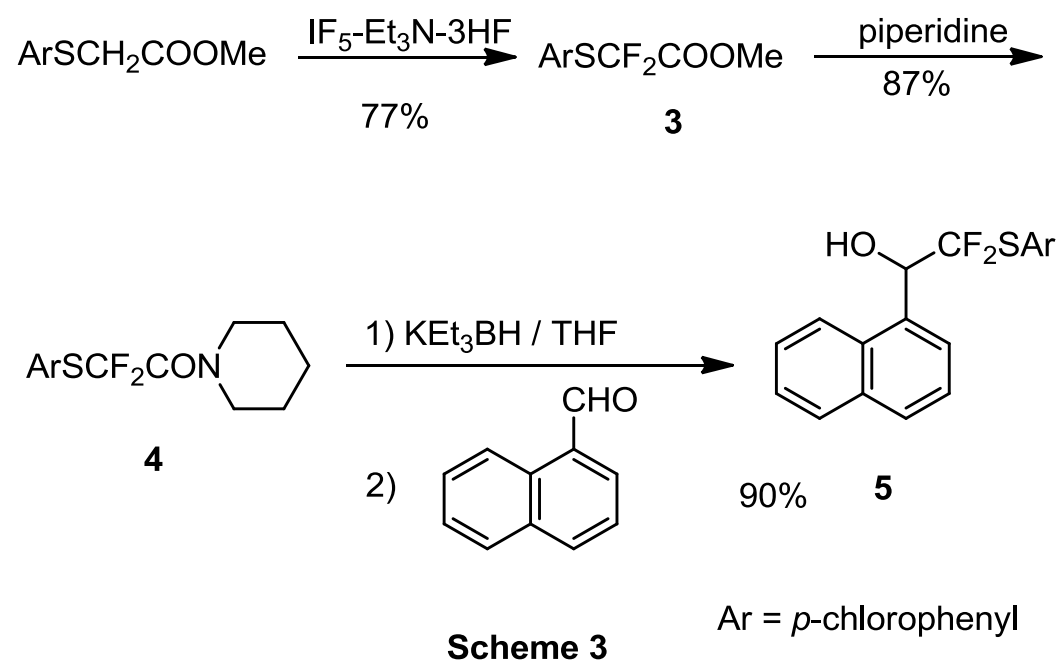

Methyl 2,2,3,3-tetrafluoro-3-phenylpropionate (7) was prepared from methyl 2-(arylsulfanyl)propionate in $74 \%$ yield by using our recently developed method, ${ }^{11}$ and the resulting 7 was converted to the amide (8). ${ }^{7}$ Although the reaction of $\mathbf{8}$ with aldehyde was sluggish, the expected polyfluoroalkylated product (9) was obtained in $60 \%$ yield by performing the reaction at $40{ }^{\circ} \mathrm{C}$ for $24 \mathrm{~h}$ using 4 equivalents of 8 and $\mathrm{KEt}_{3} \mathrm{BH}$ to the aldehyde (Scheme 4).
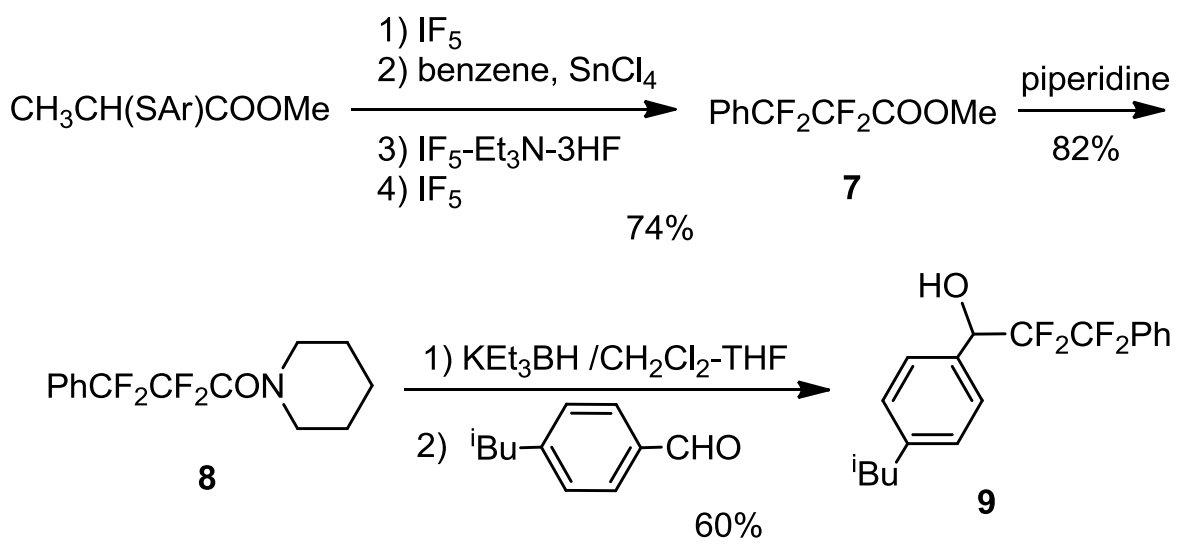

Scheme $4 \quad \operatorname{Ar}=p$-chlorophenyl

\section{EXPERIMENTAL}

\subsection{General}

The melting points were measured with a Yanagimoto micro melting-point apparatus. The IR spectra were recorded using a JASCO FT/IR-410. The ${ }^{1} \mathrm{H}$ NMR (400 MHz) spectra, ${ }^{19} \mathrm{~F}$ NMR (376 MHz) spectra, and ${ }^{13} \mathrm{C}$ NMR $(100 \mathrm{MHz})$ were recorded in $\mathrm{CDCl}_{3}$ on a JEOL JNM-A400II FT NMR and the chemical 
shift, $\delta$, is referred to TMS $\left({ }^{1} \mathrm{H},{ }^{13} \mathrm{C}\right)$ and $\mathrm{CFCl}_{3}\left({ }^{19} \mathrm{~F}\right)$, respectively. The EI-high-resolution mass spectra were measured on a JEOL JMS-700TZ. $\mathrm{KBEt}_{3} \mathrm{H}$ (1.0 M in THF) was purchased from Aldrich Chemica and $\mathrm{KB}\left(\mathrm{OPr}^{\mathrm{i}}\right)_{3} \mathrm{H}$ was prepared from $\mathrm{B}\left(\mathrm{OPr}^{\mathrm{i}}\right)_{3}$ and $\mathrm{KH}$ according to the literature. ${ }^{12} \mathrm{IF}_{5}$ in a stainless-steel cylinder was supplied by Asahi Glass Co., Ltd. $\mathrm{IF}_{5}$ was transferred through a Teflon ${ }^{\mathrm{TM}}$ tube into a Teflon ${ }^{\mathrm{TM}}$ FEP bottle from the cylinder under an $\mathrm{N}_{2}$ atmosphere. $\mathrm{IF}_{5}$ was transferred quickly from the bottle to the reaction vessel made of Teflon ${ }^{\mathrm{TM}} \mathrm{FEP}$ in open air. $\mathrm{IF}_{5}$ decomposes in air emitting HF fume, and, therefore, it should be carefully handled in a bench hood with rubber-gloved hands. ${ }^{11}$ 2,2,3,3,4,4,5,5-Octafluoropentanol was donated from Daikin Industries, Ltd.

\subsection{Preparation of $N$-(polyfluoroalkanoyl)piperidine}

$N$-(Polyfluoroalkanoyl)piperidines were prepared from the corresponding ethyl or methyl polyfluoroalkanoate with piperidine according to the literature. ${ }^{7}$

\subsection{1. $N$-(Trifluoroacetyl)piperidine (1a)}

IR (neat) 2945, 1691, 1193, $1128 \mathrm{~cm}^{-1} .{ }^{1} \mathrm{H}$ NMR (400MHz, $\left.\mathrm{CDCl}_{3}\right) \delta 1.65-1.71(6 \mathrm{H}, \mathrm{m}), 3.54-3.63(4 \mathrm{H}$, $\mathrm{m}) .{ }^{13} \mathrm{C} \mathrm{NMR}\left(100 \mathrm{MHz}, \mathrm{CDCl}_{3}\right) \delta 23.8,25.1,26.0,44.2,46.5\left(\mathrm{t},{ }^{4} J_{C-F}=3.5 \mathrm{~Hz}\right), 116.4\left(\mathrm{t},{ }^{1} J_{C-F}=287.8\right.$ $\mathrm{Hz}), 154.9\left(\mathrm{t},{ }^{2} J_{C-F}=35.3 \mathrm{~Hz}\right) .{ }^{19} \mathrm{~F}$ NMR $\left(373 \mathrm{MHz}, \mathrm{CDCl}_{3}\right) \delta-69.49$ (3F, s) ( lit. $\left.^{13}-68.02\right)$.

\subsection{2. $N$-(2,2,3,3,3-Pentafluoropropanoyl)piperidine (1b)}

IR (neat) 2947, 1684, $1175 \mathrm{~cm}^{-1} .{ }^{1} \mathrm{H}$ NMR (400MHz, $\left.\mathrm{CDCl}_{3}\right) \delta 1.64-1.71(6 \mathrm{H}, \mathrm{m}), 3.61$ (4H, brs). ${ }^{13} \mathrm{C}$ NMR $\left(100 \mathrm{MHz}, \mathrm{CDCl}_{3}\right) \delta 23.7,25.0,26.0,44.0,46.2,108.3\left(\mathrm{tq},{ }^{1} J_{C-F}=270.6 \mathrm{~Hz},{ }^{2} J_{C-F}=36.0 \mathrm{~Hz}\right)$, $117.9\left(\mathrm{tq},{ }^{2} J_{C-F}=34.4 \mathrm{~Hz},{ }^{1} J_{C-F}=285.6 \mathrm{~Hz}\right), 155.6\left(\mathrm{t},{ }^{2} J_{C-F}=24.8 \mathrm{~Hz}\right) .{ }^{19} \mathrm{~F} \mathrm{NMR}\left(373 \mathrm{MHz}, \mathrm{CDCl}_{3}\right) \delta$ $-82.77(3 \mathrm{~F}, \mathrm{~s}),-115.44(2 \mathrm{~F}, \mathrm{~s})$. HRMS (EI) calcd for $\mathrm{C}_{8} \mathrm{H}_{10} \mathrm{~F}_{5} \mathrm{NO}\left(\mathrm{M}^{+}\right)$231.06825, found 231.06888.

\subsection{3. $N$-(2,2,3,3,4,4,5,5-Octafluoropentanoyl)piperidine (1c)}

Ethyl 2,2,3,3,4,4,5,5-octafluoropentanoate was prepared from 2,2,3,3,4,4,5,5-octafluoropentanol according to the literature, ${ }^{8,9}$ and then converted to 1c according to the literature. ${ }^{7}$

IR (neat) 2950, 1682, 1450, $1166 \mathrm{~cm}^{-1} .{ }^{1} \mathrm{H}$ NMR (400MHz, $\left.\mathrm{CDCl}_{3}\right) \delta 1.65-1.71(6 \mathrm{H}, \mathrm{m}), 3.61-3.63(4 \mathrm{H}$, $\mathrm{m}), 6.33(1 \mathrm{H}, \mathrm{tt}, J=52.3,5.7 \mathrm{~Hz}) .{ }^{13} \mathrm{C} \mathrm{NMR}\left(100 \mathrm{MHz}, \mathrm{CDCl}_{3}\right) \delta 23.7,25.1,26.0,44.5,46.5\left(\mathrm{t},{ }^{4} J_{C-F}=\right.$ $6.3 \mathrm{~Hz}), 105.0-114.1(4 \mathrm{C}, \mathrm{m}), 156.3\left(\mathrm{t},{ }^{2} J_{C-F}=24.6 \mathrm{~Hz}\right) .{ }^{19} \mathrm{~F}$ NMR $\left(373 \mathrm{MHz}, \mathrm{CDCl}_{3}\right) \delta-112.13(2 \mathrm{~F}, \mathrm{t}, J=$ $9.0 \mathrm{~Hz}),-124.50$ to $-124.56(2 \mathrm{~F}, \mathrm{~m}),-129.02$ to $-129.12(2 \mathrm{~F}, \mathrm{~m}),-138.17(2 \mathrm{~F}, \mathrm{dm}, J=52.0 \mathrm{~Hz})$. HRMS (EI) calcd for $\mathrm{C}_{10} \mathrm{H}_{11} \mathrm{~F}_{8} \mathrm{NO}\left(\mathrm{M}^{+}\right)$313.07129, found 313.07058.

\subsection{4. $N$-2-\{(4-Chlorophenyl)sulfanyl\}-2,2-difluoroacetylpiperidine (4)}

Methyl 2-\{(4-chlorophenyl)sulfanyl $\}$-2,2-difluoroacetate (3) was prepared from methyl 2-\{(4-Chlorophenyl)sulfanyl $\}$ acetate according to the literature, ${ }^{10}$ and converted to $\mathbf{4}$ according to the literature. ${ }^{7}$ White solid. Mp 77-79 ${ }^{\circ} \mathrm{C}$. IR (KBr) 2935, 1671, $1038 \mathrm{~cm}^{-1} .{ }^{1} \mathrm{H} \mathrm{NMR}\left(400 \mathrm{MHz}, \mathrm{CDCl}_{3}\right) \delta$ 1.62-1.69 (6H, m), 3.60-3.64 (4H, m), $7.37(2 \mathrm{H}, \mathrm{d}, J=8.3 \mathrm{~Hz}), 7.56(2 \mathrm{H}, \mathrm{d}, J=8.3 \mathrm{~Hz}) .{ }^{13} \mathrm{C} \mathrm{NMR}$ 
$\left(100 \mathrm{MHz}, \mathrm{CDCl}_{3}\right) \delta 24.2,25.5,26.3,44.8,47.0\left(\mathrm{t},{ }^{4} J_{C-F}=5.0 \mathrm{~Hz}\right), 124.2,124.6\left(\mathrm{t},{ }^{1} J_{C-F}=291.4 \mathrm{~Hz}\right)$, $129.2(2 \mathrm{C}), 136.8,137.9(2 \mathrm{C}), 159.2\left(\mathrm{t},{ }^{2} J_{C-F}=26.2 \mathrm{~Hz}\right) .{ }^{19} \mathrm{~F}$ NMR $\left(373 \mathrm{MHz}, \mathrm{CDCl}_{3}\right) \delta-73.52(2 \mathrm{~F}, \mathrm{~s})$. HRMS (EI) calcd for $\mathrm{C}_{13} \mathrm{H}_{14} \mathrm{Cl} \mathrm{F}_{2} \mathrm{NOS}\left(\mathrm{M}^{+}\right)$305.04494, found 305.04527 .

\subsection{5. $N$-(2,2,3,3-Tetrafluoro-3-phenylpopanoyl)piperidine (8)}

Methyl 3-phenyl-2,2,3,3-tetrafluoropropionate (7) was prepared from methyl 2-\{(4-chlorophenyl)sulfanyl $\}$ propionate in three steps according to the literature, ${ }^{11}$ and converted to $\mathbf{8}$ by the reaction with piperidine. ${ }^{7}$ IR (neat) $2941,1675,1452,1294,1120 \mathrm{~cm}^{-1} .{ }^{1} \mathrm{H} \mathrm{NMR}\left(400 \mathrm{MHz}, \mathrm{CDCl}_{3}\right) \delta$ 1.63-1.68 (6H, m), 3.59-3.64 (4H, m), 7.45-7.52 (3H, m), $7.62(2 \mathrm{H}, \mathrm{d}, J=7.5 \mathrm{~Hz}) .{ }^{13} \mathrm{C}$ NMR $(100 \mathrm{MHz}$, $\left.\mathrm{CDCl}_{3}\right) \delta 24.2,25.5,26.4,44.8,46.9-47.1(\mathrm{~m}), 111.6\left(\mathrm{tt},{ }^{1} J_{C-F}=264.6 \mathrm{~Hz},{ }^{2} J_{C-F}=37.4 \mathrm{~Hz}\right), 116.1(\mathrm{tt}$, $\left.{ }^{1} J_{C-F}=253.5 \mathrm{~Hz},{ }^{2} J_{C-F}=31.5 \mathrm{~Hz}\right), 126.8\left(\mathrm{tt},{ }^{3} J_{C-F}=6.5 \mathrm{~Hz},{ }^{4} J_{C-F}=1.5 \mathrm{~Hz}\right), 128.1(2 \mathrm{C}), 130.3\left(\mathrm{t},{ }^{2} J_{C-F}=\right.$ $24.3 \mathrm{~Hz}), 131.1\left(2 \mathrm{C}, \mathrm{t},{ }^{4} J_{C-F}=1.7 \mathrm{~Hz}\right), 157.7\left(\mathrm{t},{ }^{2} J_{C-F}=26.0 \mathrm{~Hz}\right) \cdot{ }^{19} \mathrm{~F}$ NMR $\left(373 \mathrm{MHz}, \mathrm{CDCl}_{3}\right) \delta-110.91$ $(2 \mathrm{~F}, \mathrm{~s}),-111.62(2 \mathrm{~F}, \mathrm{~s})$. HRMS (EI) calcd for $\mathrm{C}_{14} \mathrm{H}_{15} \mathrm{~F}_{4} \mathrm{NO}\left(\mathrm{M}^{+}\right)$289.10898, found 289.10820.

\subsection{Perfluoroalkylation of carbonyl compounds}

\section{4,3,1. 2,2,2-Trifluoro-1-phenylethanol (2a)}

To a THF solution $(3 \mathrm{~mL})$ of $\mathbf{1 a}(181 \mathrm{mg}, 1 \mathrm{mmol})$ were added a $1.0 \mathrm{M}$ THF solution of Et ${ }_{3} \mathrm{BHK}(1 \mathrm{~mL}, 1$ mmol) and benzaldehyde (53 mg, $0.5 \mathrm{mmol}$ ) successively at room temperature under $\mathrm{N}_{2}$ atmosphere. The mixture was stirred at $50{ }^{\circ} \mathrm{C}$ for $24 \mathrm{~h}$ and then $30 \%$ aqueous $\mathrm{H}_{2} \mathrm{O}_{2}(2 \mathrm{~mL})$ was added at $0{ }^{\circ} \mathrm{C}$. After stirring for $1 \mathrm{~h}$, the mixture was extracted with ether $(30 \mathrm{~mL} \mathrm{X} \mathrm{3)}$. The combined organic phase was dried over $\mathrm{MgSO}_{4}$ and the yield of $\mathbf{2 a}$ was determined by ${ }^{19} \mathrm{~F}$ NMR using fluorobenzene as internal standard (99\%). Pure 2a was obtained by column chromatography (silica gel/ $\mathrm{CH}_{2} \mathrm{Cl}_{2}$ :hexane $=4: 1$ ). IR (neat) 3397 , 1267, $1127 \mathrm{~cm}^{-1} .{ }^{1} \mathrm{H}$ NMR $\left(400 \mathrm{MHz}, \mathrm{CDCl}_{3}\right) \delta 5.01-5.06(1 \mathrm{H}, \mathrm{m}), 7.41-7.49(5 \mathrm{H}, \mathrm{m}) .{ }^{13} \mathrm{C} \mathrm{NMR}$ $\left(100 \mathrm{MHz}, \mathrm{CDCl}_{3}\right) \delta 72.8\left(\mathrm{q},{ }^{2} J_{C-F}=31.9 \mathrm{~Hz}\right), 124.3\left(\mathrm{q},{ }^{1} J_{C-F}=282.3 \mathrm{~Hz}\right), 127.4,128.6(2 \mathrm{C}), 129.6(2 \mathrm{C})$, $133.9\left(\mathrm{q},{ }^{3} J_{C-F}=0.9 \mathrm{~Hz}\right) .{ }^{19} \mathrm{~F}$ NMR $\left(373 \mathrm{MHz}, \mathrm{CDCl}_{3}\right) \delta-78.99(3 \mathrm{~F}, \mathrm{~d}, J=7.1 \mathrm{~Hz})\left\{\right.$ lit. $^{14}-78.77(\mathrm{~d}, J=7.6$ $\mathrm{Hz})\}$.

\section{4,2,2. 1-(4-Bromophenyl)-2,2,2-trifluoroethanol (2b)}

IR (neat) 3397, 1492, 1268, 1173, $1130 \mathrm{~cm}^{-1} .{ }^{1} \mathrm{H}$ NMR (400MHz, $\mathrm{CDCl}_{3}$ ) $\delta$ 4.98-5.03 (1H, m), 7.35-7.44 $(2 \mathrm{H}, \mathrm{m}), 7.54-7.56(2 \mathrm{H}, \mathrm{m}) .{ }^{13} \mathrm{C} \mathrm{NMR}\left(100 \mathrm{MHz}, \mathrm{CDCl}_{3}\right) \delta 72.2\left(\mathrm{q},{ }^{2} J_{C-F}=32.2 \mathrm{~Hz}\right), 123.8,123.9$ (q, $\left.{ }^{1} J_{C-F}=282.1 \mathrm{~Hz}\right), 129.0(2 \mathrm{C}), 131.8(2 \mathrm{C}), 132.7 .{ }^{19} \mathrm{~F} \mathrm{NMR}\left(373 \mathrm{MHz}, \mathrm{CDCl}_{3}\right) \delta-79.15(3 \mathrm{~F}, \mathrm{~d}, J=7.1 \mathrm{~Hz})$ $\left\{\right.$ lit. $\left.^{14}-78.94(\mathrm{~d}, J=6.8 \mathrm{~Hz})\right\}$.

\section{4,2,3. (E)-1,1,1-Trifluoro-4-phenylbut-3-en-2-ol (2c)}

White solid. Mp 42-43 ${ }^{\circ} \mathrm{C}$ (lit. ${ }^{15} 42-43{ }^{\circ} \mathrm{C}$ ). IR (KBr) 3315, 2924, 1453, $1371 \mathrm{~cm}^{-1} .{ }^{1} \mathrm{H}$ NMR (400MHz, $\left.\mathrm{CDCl}_{3}\right) \delta 4.61-4.68(1 \mathrm{H}, \mathrm{m}), 6.21(1 \mathrm{H}, \mathrm{dd}, J=15.9,6.5 \mathrm{~Hz}), 6.86(1 \mathrm{H}, \mathrm{d}, J=15.9 \mathrm{~Hz}), 7.29-7.44(5 \mathrm{H}, \mathrm{m})$.

${ }^{13} \mathrm{C} \mathrm{NMR}\left(100 \mathrm{MHz}, \mathrm{CDCl}_{3}\right) \delta 71.6\left(\mathrm{q},{ }^{2} J_{C-F}=32.4 \mathrm{~Hz}\right), 120.5(\mathrm{q}, J=1.9 \mathrm{~Hz}), 124.2(\mathrm{q}, J=281.8 \mathrm{~Hz})$, 
126.9 (2C), 128.7 (2C), 128.8, 135.3, 136.4. ${ }^{19} \mathrm{~F} \mathrm{NMR}\left(373 \mathrm{MHz}, \mathrm{CDCl}_{3}\right) \delta-79.70(3 \mathrm{~F}, \mathrm{~d}, J=7.2 \mathrm{~Hz})$ $\left\{1 \mathrm{it}^{15}-79.7(\mathrm{~d}, J=6.4 \mathrm{~Hz})\right\}$.

\section{4,2,4. 2,2,2-Trifluoro-1-(naphthalen-1-yl)ethanol (2d)}

IR (neat) 3399, 2941, 1265, 1168, $1126 \mathrm{~cm}^{-1} .{ }^{1} \mathrm{H} \mathrm{NMR}\left(400 \mathrm{MHz}, \mathrm{CDCl}_{3}\right) \delta 2.69(1 \mathrm{H}, \mathrm{d}, J=4.5 \mathrm{~Hz})$, 5.88-5.94 (1H, m), 7.51-7.60 (3H, m), $7.84(1 \mathrm{H}, \mathrm{d}, J=7.3 \mathrm{~Hz}), 7.90-7.93(2 \mathrm{H}, \mathrm{m}), 8.08(1 \mathrm{H}, \mathrm{d}, J=8.4$ $\mathrm{Hz}) .{ }^{13} \mathrm{C} \mathrm{NMR}\left(100 \mathrm{MHz}, \mathrm{CDCl}_{3}\right) \delta 68.8\left(\mathrm{q},{ }^{2} J_{C-F}=32.2 \mathrm{~Hz}\right), 122.7\left(\mathrm{q},{ }^{4} J_{C-F}=0.9 \mathrm{~Hz}\right), 124.6\left(\mathrm{q},{ }^{1} J_{C-F}=\right.$ $282.5 \mathrm{~Hz}), 125.1,125.7\left(\mathrm{q},{ }^{3} J_{C-F}=1.2 \mathrm{~Hz}\right), 125.9,126.8,129.0,129.8130 .1,131.0,133.6 .{ }^{19} \mathrm{~F}$ NMR $\left(373 \mathrm{MHz}, \mathrm{CDCl}_{3}\right) \delta-77.48(3 \mathrm{~F}, \mathrm{~d}, J=7.2 \mathrm{~Hz})\left\{\right.$ lit. $\left.^{14}-77.25(\mathrm{~d}, J=6.5 \mathrm{~Hz})\right\}$.

\section{4,2,5. 2,2,2-Trifluoro-1-(furan-2-yl)ethanol (2e)}

IR (neat) 3399, 1669, 1504, $1152 \mathrm{~cm}^{-1} .{ }^{1} \mathrm{H}$ NMR (400MHz, $\left.\mathrm{CDCl}_{3}\right) \delta 2.59(1 \mathrm{H}, \mathrm{d}, J=7.2 \mathrm{~Hz}), 5.03-5.10$ $(1 \mathrm{H}, \mathrm{m}), 6.53-6.44(1 \mathrm{H}, \mathrm{m}), 6.54(1 \mathrm{H}, \mathrm{d}, J=3.2 \mathrm{~Hz}), 7.47-7.48(1 \mathrm{H}, \mathrm{m}) .{ }^{13} \mathrm{C} \mathrm{NMR}\left(100 \mathrm{MHz}, \mathrm{CDCl}_{3}\right) \delta$ $67.1\left(\mathrm{q},{ }^{2} J_{C-F}=34.3 \mathrm{~Hz}\right), 110.1\left(\mathrm{q},{ }^{4} J_{C-F}=0.9 \mathrm{~Hz}\right), 110.7,123.4\left(\mathrm{q},{ }^{1} J_{C-F}=282.1 \mathrm{~Hz}\right), 143.6,147.1(\mathrm{q}$, $\left.{ }^{3} J_{C-F}=1.6 \mathrm{~Hz}\right) .{ }^{19} \mathrm{~F}$ NMR $\left(373 \mathrm{MHz}, \mathrm{CDCl}_{3}\right) \delta-78.59(3 \mathrm{~F}, \mathrm{~d}, J=7.2 \mathrm{~Hz})\left\{\mathrm{lit}^{3 \mathrm{c}}-78.42(\mathrm{~d}, J=6.4 \mathrm{~Hz})\right\}$.

\section{4,2,6. 1-(1-Adamantan-1-yl)-2,2,2-trifluoroethanol (2f)}

Yellow solid. Mp 47-49 ${ }^{\circ} \mathrm{C}$. IR (KBr) 3422, 2907, 2851, 1263, 1167, $1120 \mathrm{~cm}^{-1}$. ${ }^{1} \mathrm{H}$ NMR (400MHz, $\left.\mathrm{CDCl}_{3}\right) \delta 1.68-1.79(11 \mathrm{H}, \mathrm{m}), 2.02(3 \mathrm{H}, \mathrm{brs}), 3.44(1 \mathrm{H}, \mathrm{q}, J=8.3 \mathrm{~Hz}) .{ }^{13} \mathrm{C} \mathrm{NMR}\left(100 \mathrm{MHz}, \mathrm{CDCl}_{3}\right) \delta$ $28.0(3 \mathrm{C}), 35.7(3 \mathrm{C}), 36.7(3 \mathrm{C}), 37.6\left(\mathrm{q},{ }^{4} J_{C-F}=1.8 \mathrm{~Hz}\right), 77.5\left(\mathrm{q},{ }^{2} J_{C-F}=27.9 \mathrm{~Hz}\right), 125.6\left(\mathrm{q},{ }^{1} J_{C-F}=285.1\right.$ $\mathrm{Hz}) .{ }^{19} \mathrm{~F}$ NMR $\left(373 \mathrm{MHz}, \mathrm{CDCl}_{3}\right) \delta-71.55(3 \mathrm{~F}, \mathrm{~d}, J=8.8 \mathrm{~Hz})$. HRMS (EI) calcd for $\mathrm{C}_{12} \mathrm{H}_{16} \mathrm{~F}_{3} \mathrm{O}\left(\mathrm{M}^{+}\right)$ 233.11587, found 233.11668.

\section{4,2,7. 1-Cyclohexyl-2,2,2-trifluoroethanol (2g)}

IR (neat) 3399, 3055, 1476, 1162, $1060 \mathrm{~cm}^{-1} .{ }^{1} \mathrm{H}$ NMR (400MHz, $\left.\mathrm{CDCl}_{3}\right) \delta 1.10-1.34$ (5H, m), 1.67-2.02 $(6 \mathrm{H}, \mathrm{m}), 3.68-3.77(1 \mathrm{H}, \mathrm{m}) .{ }^{13} \mathrm{C}$ NMR $\left(100 \mathrm{MHz}, \mathrm{CDCl}_{3}\right) \delta 25.7,25.9,26.0,26.7\left(\mathrm{q},{ }^{4} J_{C-F}=1.0 \mathrm{~Hz}\right), 29.2$ $\left(\mathrm{q},{ }^{3} J_{C-F}=1.2 \mathrm{~Hz}\right), 38.1\left(\mathrm{q},{ }^{4} J_{C-F}=1.0 \mathrm{~Hz}\right), 74.3\left(\mathrm{q},{ }^{2} J_{C-F}=29.1 \mathrm{~Hz}\right), 125.3\left(\mathrm{q},{ }^{1} J_{C-F}=283.3 \mathrm{~Hz}\right) .{ }^{19} \mathrm{~F}$ NMR $\left(373 \mathrm{MHz}, \mathrm{CDCl}_{3}\right) \delta-76.2(3 \mathrm{~F}, \mathrm{~d}, J=7.1 \mathrm{~Hz})\left\{\right.$ lit. $\left.^{3 \mathrm{c}}-75.98(\mathrm{~d}, J=7.5 \mathrm{~Hz})\right\}$.

\section{4,2,8. 2,2,2-Trifluoro-1,1-diphenylethanol (2h)}

IR (neat) 3456, 3063, 1714, $1155 \mathrm{~cm}^{-1} .{ }^{1} \mathrm{H}$ NMR (400MHz, $\left.\mathrm{CDCl}_{3}\right) \delta$ 7.34-7.38 (6H, m), 7.48-7.50 (4H, m). ${ }^{13} \mathrm{C}$ NMR $\left(100 \mathrm{MHz}, \mathrm{CDCl}_{3}\right) \delta 79.36\left(\mathrm{q},{ }^{2} J_{C-F}=28.4 \mathrm{~Hz}\right), 125.3\left(\mathrm{q},{ }^{1} J_{C-F}=286.1 \mathrm{~Hz}\right), 127.4(2 \mathrm{C}, \mathrm{q}$, $\left.{ }^{3} J_{C-F}=1.4 \mathrm{~Hz}\right), 128.2(4 \mathrm{C}), 128.6(4 \mathrm{C}), 139.4(2 \mathrm{C}) .{ }^{19} \mathrm{~F} \mathrm{NMR}\left(373 \mathrm{MHz}, \mathrm{CDCl}_{3}\right) \delta-74.91$ (3F, s) (lit. ${ }^{16}$ $-74.21)$.

\section{4,2,9. 2,2,3,3,3-Pentafluoro-1-(naphthalen-1-yl)propan-1-ol (2i)}

The reaction was carried out as in the case of $\mathbf{2 a}$ using $\mathbf{1 b}$ and 1-naphthaldehyde instead of $\mathbf{1 a}$ and benzaldehyde, and $\mathbf{2} \mathbf{i}$ was isolated by column chromatography (silica gel, hexane: $\mathrm{CH}_{2} \mathrm{Cl}_{2}=4: 1$ ) in $89 \%$ yield. White solid. Mp 44-46 ${ }^{\circ} \mathrm{C}$. IR (KBr) 3465, 3060, 1185, 1135, $1028 \mathrm{~cm}^{-1}$. ${ }^{1} \mathrm{H}$ NMR $(400 \mathrm{MHz}$, 
$\left.\mathrm{CDCl}_{3}\right) \delta 2.49(1 \mathrm{H}, \mathrm{d}, J=4.1 \mathrm{~Hz}), 6.06(1 \mathrm{H}, \mathrm{dt}, J=18.3,4.5 \mathrm{~Hz}), 7.52-7.61(3 \mathrm{H}, \mathrm{m}), 7.84(1 \mathrm{H}, \mathrm{d}, J=7.2$ $\mathrm{Hz}), 7.90-7.95(2 \mathrm{H}, \mathrm{m}), 8.03(1 \mathrm{H}, \mathrm{d}, J=8.3 \mathrm{~Hz}) .{ }^{13} \mathrm{C} \mathrm{NMR}\left(100 \mathrm{MHz}, \mathrm{CDCl}_{3}\right) \delta 67.4\left(\mathrm{dd},{ }^{2} J_{\mathrm{C}-F}=26.3 \mathrm{~Hz}\right.$, $\left.{ }^{2} J_{C-F}=21.9 \mathrm{~Hz}\right), 113.3\left(\mathrm{ddq},{ }^{1} J_{C-F}=262.2 \mathrm{~Hz},{ }^{1} J_{C-F}=254.8 \mathrm{~Hz},{ }^{2} J_{C-F}=35.7 \mathrm{~Hz}\right), 119.3\left(\mathrm{ddq},{ }^{2} J_{C-F}=36.7\right.$

$\left.\mathrm{Hz},{ }^{2} J_{C-F}=35.1 \mathrm{~Hz},{ }^{1} J_{C-F}=287.1 \mathrm{~Hz}\right), 122.6\left(\mathrm{dd},{ }^{3} J_{C-F}=2.4 \mathrm{~Hz},{ }^{3} J_{C-F}=1.4 \mathrm{~Hz}\right), 125.1,125.9,126.4$, 126.8, 129.0, 130.0, 130.2, 131.2, 133.6. ${ }^{19} \mathrm{~F} \mathrm{NMR}\left(373 \mathrm{MHz}, \mathrm{CDCl}_{3}\right) \delta-82.19(3 \mathrm{~F}, \mathrm{~s}),-120.22(1 \mathrm{~F}, \mathrm{~d}, J=$ $277.6 \mathrm{~Hz}),-130.51(1 \mathrm{~F}, \mathrm{dd}, J=276.7,17.9 \mathrm{~Hz})\left\{\right.$ lit. $\left.^{17}-81.54(\mathrm{~m}, 3 \mathrm{~F})\right\},-118.15(\mathrm{dd}, J=290.4,20.7 \mathrm{~Hz}$, $1 \mathrm{~F}),-130.24(\mathrm{dd}, J=290.4,20.7 \mathrm{~Hz}, 1 \mathrm{~F})$.

\section{4,2,9. 2,2,3,3,4,4,5,5-Octafluoro-1-(naphthalen-1-yl)pentan-1-ol (2j)}

To 1c $(626 \mathrm{mg}, 2 \mathrm{mmol})$ were added a $1.0 \mathrm{M}$ THF solution of $\mathrm{Et}_{3} \mathrm{BHK}(2 \mathrm{~mL}, 2 \mathrm{mmol})$ and 1-naphthaldehyde $(78 \mathrm{mg}, 0.5 \mathrm{mmol})$ successively at room temperature under $\mathrm{N}_{2}$ atmosphere. Then a volatile part was removed under reduced pressure, and the resulting viscous mixture was stirred at $40{ }^{\circ} \mathrm{C}$ for $24 \mathrm{~h}$. Then, $30 \%$ aqueous $\mathrm{H}_{2} \mathrm{O}_{2}(2 \mathrm{~mL})$ was added at $0{ }^{\circ} \mathrm{C}$, and after stirring for $1 \mathrm{~h}$, the mixture was extracted with ether (30 mL X 3). The combined organic phase was dried over $\mathrm{MgSO}_{4}$ and $\mathbf{2 j}$ was isolated by column chromatography (silica gel/ $\mathrm{CH}_{2} \mathrm{Cl}_{2}$ :hexane $=1: 4$ ) in $61 \%$ yield. White solid. Mp 75-77 ${ }^{\circ} \mathrm{C}$. IR (KBr) 3398, 1175, 1124, $1041 \mathrm{~cm}^{-1} .{ }^{1} \mathrm{H}$ NMR (400MHz, $\left.\mathrm{CDCl}_{3}\right) \delta 2.57(1 \mathrm{H}, \mathrm{s}), 6.11(1 \mathrm{H}, \mathrm{tt}$, $J=52.1,5.5 \mathrm{~Hz}), 7.51-7.60(3 \mathrm{H}, \mathrm{m}), 7.84(1 \mathrm{H}, \mathrm{d}, J=7.3 \mathrm{~Hz}), 7.90-7.94(2 \mathrm{H}, \mathrm{m}), 8.00(1 \mathrm{H}, \mathrm{d}, J=8.4 \mathrm{~Hz})$. ${ }^{13} \mathrm{C} \mathrm{NMR}\left(100 \mathrm{MHz}, \mathrm{CDCl}_{3}\right) \delta 67.3\left(\mathrm{dd},{ }^{2} J_{C-F}=21.8 \mathrm{~Hz},{ }^{2} J_{C-F}=30.5 \mathrm{~Hz}\right), 104.8-118.3(4 \mathrm{C}, \mathrm{m}), 122.6$, 125.1, 125.9, 126.5, 126.9, 129.0, 130.1, 130.2, 131.3, 133.5. ${ }^{19} \mathrm{~F}$ NMR $\left(373 \mathrm{MHz}, \mathrm{CDCl}_{3}\right) \delta-116.37(1 \mathrm{~F}$, $\mathrm{d}, J=286.6 \mathrm{~Hz}),-123.50$ to $-125.38(2 \mathrm{~F}, \mathrm{~m}),-127.40(1 \mathrm{~F}, \mathrm{~d}, J=284.8 \mathrm{~Hz}),-129.7$ to $-131.90(2 \mathrm{~F}, \mathrm{~m})$, -136.94 to -138.82 (2F, m). HRMS (ESI) calcd for $\mathrm{C}_{15} \mathrm{H}_{9} \mathrm{C} \mathrm{F}_{8} \mathrm{O}\left(\mathrm{M}^{+}\right)$357.05311, found 357.05331.

\section{4,2,10. 2-\{(4-Chlorophenyl)sulfanyl\}-2,2-difluoro-1-(naphthalen-1-yl)ethanol (5)}

The reaction was carried out as in the case of $\mathbf{2 i}$ using $\mathbf{4}$ instead of $\mathbf{1 b}$, and $\mathbf{5}$ was isolated in $90 \%$ yield by column chromatography (silica gel, hexane: $\mathrm{CH}_{2} \mathrm{Cl}_{2}=4: 1$ ). Oil. IR (neat) 3434, 2931, 2857, 1276, 1166 $\mathrm{cm}^{-1} .{ }^{1} \mathrm{H}$ NMR $\left(400 \mathrm{MHz}, \mathrm{CDCl}_{3}\right) \delta$ 5.89-5.94 (1H, m), 7.26-7.33 (2H, m), 7.48-7.57 (5H, m), 7.88-7.91 $(3 \mathrm{H}, \mathrm{m}), 8.01(1 \mathrm{H}, \mathrm{d}, J=8.1 \mathrm{~Hz}) .{ }^{13} \mathrm{C} \mathrm{NMR}\left(100 \mathrm{MHz}, \mathrm{CDCl}_{3}\right) \delta 72.0\left(\mathrm{dd},{ }^{2} J_{C-F}=27.9 \mathrm{~Hz},{ }^{2} J_{C-F}=26.2\right.$ $\mathrm{Hz}), 123.1,124.2,125.1,125.7,126.1,126.5,128.9,129.2(2 \mathrm{C}), 129.2$ (t, $\left.{ }^{1} J_{C-F}=286.5 \mathrm{~Hz}\right), 129.9(2 \mathrm{C})$, 131.2, 131.3, 133.6, 136.5, 137.6. ${ }^{19} \mathrm{~F}$ NMR $\left(373 \mathrm{MHz}, \mathrm{CDCl}_{3}\right) \delta-79.76(1 \mathrm{~F}, \mathrm{dd}, J=207.8,7.2 \mathrm{~Hz})$, $-83.60(1 \mathrm{~F}, \mathrm{dd}, J=207.8,8.8 \mathrm{~Hz})$. HRMS (ESI) calcd for $\mathrm{C}_{18} \mathrm{H}_{13} \mathrm{Cl}_{2} \mathrm{~F}_{2} \mathrm{OS}\left(\mathrm{M}^{+}+\mathrm{Cl}\right) 385.00322$, found 385.00203

\section{4,2,11. 2,2,3,3-Tetrafluoro-1-(4-isobutylphenyl)-3-phenylpropan-1-ol (9)}

To a $\mathrm{CH}_{2} \mathrm{Cl}_{2}$ solution $(1 \mathrm{~mL})$ of $\mathbf{8}(578 \mathrm{mg}, 2 \mathrm{mmol})$ were added a $1.0 \mathrm{M}$ THF solution of $\mathrm{Et}_{3} \mathrm{BHK}(2 \mathrm{~mL}$, $2 \mathrm{mmol}$ ) and 4-Isobutylbenzaldehyde ( $81 \mathrm{mg}, 0.5 \mathrm{mmol}$ ) successively at room temperature under $\mathrm{N}_{2}$ atmosphere. The mixture was stirred at $40{ }^{\circ} \mathrm{C}$ for $24 \mathrm{~h}$ and then $30 \%$ aqueous $\mathrm{H}_{2} \mathrm{O}_{2}(2 \mathrm{~mL})$ was added at 
$0{ }^{\circ} \mathrm{C}$. After stirring for $1 \mathrm{~h}$, the mixture was extracted with ether $(30 \mathrm{~mL} \mathrm{X} \mathrm{3)}$. The combined organic phase was dried over $\mathrm{MgSO}_{4}$ and 9 was isolated by column chromatography (silica gel/AcOEt:hexane = 1:10) in $60 \%$ yield. White solid. Mp 54-55 ${ }^{\circ} \mathrm{C}$. IR (KBr) 3466, 2960, $1069 \mathrm{~cm}^{-1}$. ${ }^{1} \mathrm{H}$ NMR (400MHz, $\left.\mathrm{CDCl}_{3}\right) \delta 0.89(6 \mathrm{H}, \mathrm{d}, J=6.7 \mathrm{~Hz}), 1.80-1.91(1 \mathrm{H}, \mathrm{m}), 2.39(1 \mathrm{H}, \mathrm{d}, J=4.7 \mathrm{~Hz}), 2.47(1 \mathrm{H}, \mathrm{d}, J=7.2 \mathrm{~Hz})$, $5.14(1 \mathrm{H}, \mathrm{dt}, J=17.3,5.1 \mathrm{~Hz}), 7.15(2 \mathrm{H}, \mathrm{d}, J=8.1 \mathrm{~Hz}), 7.34(2 \mathrm{H}, \mathrm{d}, J=8.0 \mathrm{~Hz}), 7.43-7.52(3 \mathrm{H}, \mathrm{m}), 7.58$ $(2 \mathrm{H}, \mathrm{d}, J=7.4 \mathrm{~Hz}) .{ }^{13} \mathrm{C} \mathrm{NMR}\left(100 \mathrm{MHz}, \mathrm{CDCl}_{3}\right) \delta 22.3(2 \mathrm{C}), 30.1,45.1,72.0\left(\mathrm{dd},{ }^{2} J_{C-F}=28.8 \mathrm{~Hz},{ }^{2} J_{C-F}=\right.$ $22.4 \mathrm{~Hz}), 115.4\left(\mathrm{tt},{ }^{1} J_{C-F}=260.8 \mathrm{~Hz},{ }^{2} J_{C-F}=35.3 \mathrm{~Hz}\right), 117.2\left(\mathrm{tt},{ }^{1} J_{C-F}=253.0 \mathrm{~Hz},{ }^{2} J_{C-F}=34.1 \mathrm{~Hz}\right), 126.7$

$\left(\mathrm{t},{ }^{3} J_{C-F}=6.7 \mathrm{~Hz}\right), 127.8(2 \mathrm{C}), 128.2(2 \mathrm{C}), 129.1(2 \mathrm{C}), 130.9\left(2 \mathrm{C}, \mathrm{t},{ }^{2} J_{C-F}=24.3 \mathrm{~Hz}\right), 131.0\left(\mathrm{t},{ }^{4} J_{C-F}=1.4\right.$ $\mathrm{Hz}), 132.5,142.8 .{ }^{19} \mathrm{~F}$ NMR $\left(373 \mathrm{MHz}, \mathrm{CDCl}_{3}\right) \delta-109.50$ to $-110.99(2 \mathrm{~F}, \mathrm{~m}),-108.29(1 \mathrm{~F}, \mathrm{dd}, J=275.5$, $6.2 \mathrm{~Hz}),-108.29(1 \mathrm{~F}, \mathrm{dd}, J=275.5,17.6 \mathrm{~Hz})$. HRMS (EI) calcd for $\mathrm{C}_{19} \mathrm{H}_{19} \mathrm{~F}_{4} \mathrm{O}\left(\mathrm{M}^{+}\right) 339.13775$, found 339.13808 .

\section{ACKNOWLEDGEMENTS}

We are grateful to Asahi Glass Co., Ltd., and Daikin Industries, Ltd., for their donation of $\mathrm{IF}_{5}$ and 2,2,3,3,4,4,5,5-octafluoropentanol, respectively.

\section{REFERENCES (AND NOTES)}

1. As for reviews and books related to nucleophilic trifluoromethylation, see: (a) D. J. Burton and Z.-Y. Yang, Tetrahedron, 1992, 48, 189; (b) M. A. McClinton and D. A. McClinton, Tetrahedron, 1992, 48, 6555; (c) G. K. S. Prakash and A. K. Yudin, Chem. Rev., 1997, 97, 757; (d) R. P. Singh and J. M. Shreeve, Tetrahedron, 2000, 56, 7613; (e) G. K. S. Prakash and M. Mandal, J. Fluorine Chem., 2001, 112, 123; (f) B. R. Langlois and T. Billard, Synthesis, 2003, 185; (g) J.-A. Ma and D. Cahard, Chem. Rev. 2004, 104, 6119; (h) T. Umemoto, 'Fluorine-Containing Synthons: Recent Advances in Perfluoroalkylation Methodology,' ed. by V. A. Soloshonok, American Chemical Society, Washington, DC, 2005, pp. 2-15; (i) G. K. S. Prakash and J. Hu, 'Fluorine-Containing Synthons: New Nucleophilic Fluoroalkylation Chemistry,' ed. by V. A. Soloshonok, American Chemical Society, Washington, DC, 2005, pp. 16-56; (j) B. R. Langlois and T. Billard, 'Fluorine-Containing Synthons: Fluoroform, Fluoral, Trifluoroacetic and Trifluoromethanesulfinic Acids Derivatives as New Reagents for the Introduction of Polyfluorinated Moieties,' ed. by V. A. Soloshonok, American Chemical Society, Washington, DC, 2005, pp. 57-86; (k) B. R. Langlois, T. Billard, and S. Roussel, J. Fluorine Chem., 2005, 126, 173; (1) G. K. S. Prakash and J. Hu, Acc. Chem. Res., 2007, 40, 921; (m) M. Médebielle and W. R. Dolbier Jr., J. Fluorine Chem., 2008, 129, 930; (n) J. Gawronski, N. Wascinska, and J. Gajewy, Chem. Rev., 2008, 108, 5227; (o) N. Shibata, S. Mizuta, and H. Kawai, Tetrahedron: Asymmetry, 2008, 19, 2633; (p) G. Valero, X. Companyó, and R. Rios, Chem. Eur. J., 
2011, 17, 2018.

2. G. K. S. Prakash, R. Krishnamurti, and G. A. Olah, J. Am. Chem. Soc., 1989, 111, 393; R. Krishnamurti, D. R. Bellew, and G. K. S. Prakash, J. Org. Chem., 1991, 56, 984; R. P. Singh, G. Gao, R. L. Kirchmeier, and J. M. Shreeve, J. Org. Chem., 1999, 64, 2873; J. Kim and J. M. Shreeve, Org. Biomol. Chem., 2004, 2, 2728; J. J. Song, Z. Tan, J. T. Reeves, F. Gallou, N. K. Yee, and C. H. Senanayake, Org. Lett., 2005, 7, 2193; S. Mizuta, N. Shibata, S. Ogawa, H. Fujimoto, S. Nakamura, and T. Toru, Chem. Commun., 2006, 2575; V. V. Levin, A. D. Dilman, P. A. Belyakov, M. I. Struchkova, and V. A. Tartakovsky, Tetrahedron, 2011, 52, 281.

3. (a) B. Folléas, I. Marek, J.-F. Normant, and L. S. Jalmes, Tetrahedron Lett., 1998, 39, 2973; (b) J. Russell and N. Roques, Tetrahedron, 1998, 54, 13771; (c) B. Folléas, I. Marek, J.-F. Normmant, and L. S. Jalmes, Tetrahedron, 2000, 56, 275; (d) S. Large, N. Roques, and B. R. Langlois, J. Org. Chem., 2000, 65, 8848; (e) T. Billard, S. Bruns, and B. R. Langlois, Org. Lett., 2000, 2, 2101.

4. C. Mispelaere and N. Roques, Tetrahedron Lett., 1999, 40, 6411; T. Billard, B. R. Langlois, and G. Blond, Eur. J. Org. Chem., 2001, 1467.

5. Recently, trifluoromethyl anion was generated from fluoroform directly by using organo-superbase, see: H. Kawai, Z. Yuan, E. Tokunaga, and N. Shibata, Org. Biomol. Chem., 2013, 11, 1446.

6. Trifluoroacetamides were previously used to generate the trifluoromethyl anion by the reaction with base, but the trifluoromethylation successfully proceeded only in the reaction with aromatic ketones, see: L. Jablonski, J. Joubert, T. Billard, and B. R. Langlois, Synlett, 2003, 230.

7. R. E. Sammelson and J. E. Casida, J. Org. Chem., 2003, 68, 8075.

8. T. Hudlicky, R. Fan, J. W. Reed, D. R. Carver, M. Hudlicky, and E. I. Eger II, J. Fluorine Chem., 1992, 59, 9 .

9. J. Afzal, B. M. Fung, and E. A. O'rear, J. Fluorine Chem., 1987, 34, 385.

10. S. Ayuba, N. Yoneda, T. Fukuhara, and S. Hara, Bull. Chem. Soc. Jpn., 2002, 75, 1597.

11. T. Fukuhara and S. Hara, J. Org. Chem., 2010, 75, 7393.

12. H. C. Brown, J. S. Cha, B. Nazer, S.-C. Kim, S. Krishnamurthy, and C. A. Brown, J. Org. Chem., $1984,49,885$.

13. H. A. Schenck, P. W. Lenkowski, I. C. Mukherjee, S.-H. Ko, J. P. Stables, M. K. Patel, and M. L. Brown, Bioorg. Med. Chem., 2004, 12, 979.

14. M. Shi, X.-G. Liu, Y.-W. Guo, and W. Zhang, Tetrahedron, 2007, 63, 12731.

15. M. G. Gorbunova, I. I. Gerus, and V. P. Kukhar, J. Fluorine Chem., 1993, $65,25$.

16. J. R. White, G. J. Price, P. K. Plucinski, and C. G. Frost, Tetrahedron Lett., 2009, 50, 7365.

17. C. Pooput, W. R. Dobier, Jr., and M. Médebielle, J Org. Chem., 2006, 71, 3564. 